\title{
Aplicación del modelo SERVQUAL para la determinación de los factores que inciden en la satisfacción de los usuarios en los hogares infantiles de Chibolo, Magdalena
}

\section{Application of the SERVQUAL model for the determination of the factors influencing user satisfaction in children's homes in Chibolo, Magdalena}

DOI: https://doi.org/10.17981/ingecuc.14.2.2018.02

Artículo de investigación. Fecha de recepción:13/01/2018. Fecha de aceptación:12/06/2018.

\author{
Víctor Enrique Valencia Espejo \\ Universidad del Atlántico. Barranquilla (Colombia) \\ valenciaespejo@gmail.com \\ Selenia Cruz Botto iD \\ Universidad del Magdalena. Santa Marta (Colombia) \\ seleniacruzbotto@gmail.com \\ Oscar José Ospino Ayala \\ Universidad del Magdalena. Santa Marta (Colombia) \\ oscarospino2010@gmail.com
}

Para citar este artículo:

V. E. Valencia Espejo, S. Cruz Botto y O. J. Ospino Ayala, "Aplicación del modelo SERVQUAL para la determinación de los factores que inciden en la satisfacción de los usuarios en los hogares infantiles de Chibolo, Magdalena," INGE CUC, vol. 14, no. 2, pp. 19-27, 2018. DOI: http://doi.org/10.17981/ingecuc.14.2.2018.02

\section{Resumen}

Introducción- La primera infancia es el período de la vida que determina el desarrollo del individuo como persona. Existen factores que han afectado negativamente la satisfacción de los usuarios en los Hogares Comunitarios de Bienestar del ICBF, los cuales trabajan en pro de los infantes. Debido a esto, surge el modelo SERVQUAL como una solución que permite la medición de la calidad del servicio, conocer las percepciones y expectativas de los clientes e identificar los factores que afectan la calidad del servicio.

Objetivo- El presente estudio tiene como objetivo principal la determinación de los factores que inciden en la satisfacción de los usuarios en los hogares infantiles por medio de la aplicación de un modelo SERVQUAL.

Metodología- El artículo se divide en 3 fases. Estas son: recolección de información relevante al caso en estudio; evaluación de la importancia, expectativa y percepción del usuario frente a los componentes calidad; y determinación de los componentes de calidad que inciden en la satisfacción de los usuarios.

Resultados- Se logró la aplicación completa del modelo estadístico, obteniéndose que los componentes "salud y nutrición” son los que más afectan la percepción de la calidad del servicio, seguidos por los factores "familia comunidad, proceso pedagógico y talento humano", que también tienen un importante índice de incidencia.

Conclusiones- La empresa se encuentra en mejoramiento continuo; sin embargo, se hace necesario trabajar responsablemente para atender los componentes que influyen en la percepción de su servicio para la obtención de un mayor índice de calidad.

Palabras clave- Modelo SERVQUAL; calidad; satisfacción; importancia; expectativa; percepción; Hogares Comunitarios de Bienestar

\begin{abstract}
Introduction- Early childhood is the pert of life that determines the development of the individual as a person. Undoubtedly, experiences influence the development of children. This paper analyzes the factors that have affected negatively the satisfaction index of the ICBF Community Welfare Homes users of the that work in favor of the infants, due to this, the SERVQUAL model emerges as a solution that allows the measurement of the quality of the service, to know the perceptions and expectations of the customers, and to identify the factors that affect the quality of the service.
\end{abstract}

Objective- The purpose of this study is to determine the factors that affect the satisfaction of users in children's homes, through the application of a SERVQUAL model.

Methodology - The article consists of 3 phases: Collection of information relevant to the case under study, Evaluation of the importance, expectation and perception of the user against the components of quality and Determination of quality components that affect user satisfaction.

Results- The complete application of the statistical model was obtained, obtaining that the components "health and nutrition" are the ones that most affect the perception of the service quality, followed by the factors "Family community, pedagogical process and human talent" that also have an important index of incidence.

Conclusions - The company is in continuous improvement, however, it is necessary to work responsibly to attend to the components that influence the perception of its service, to obtain a higher quality index.

Keywords- SERVQUAL model; quality; satisfaction; importance; expectative; perception; Community Welfare Homes 


\section{INTRODUCCIÓN}

En Colombia, la primera infancia se ha considerado como la base del progreso y el aprendizaje. De esta manera, se reconoce que los "momentos del cuidado calificado donde se satisfacen condiciones básicas de afecto, alimentación, protección, higiene, entre otras, llevan implícitas acciones formativas, por cuanto se constituyen en espacios de intercambio y comunicación, es decir, en una experiencia pedagógica que potencia el desarrollo" [1]. Además, se concibe al cuidado y la educación inicial como una constituyente para reducir la pobreza y la desigualdad [2].

Debido a ello, hay 69.000 madres comunitarias, entre las cuales también existen algunos padres comunitarios que proveen de cuidados a los niños de todo el país. Ellos atienden a 1.770 .000 niños y niñas en la modalidad comunitaria de la educación inicial a través del servicio de Hogares Comunitarios en todas sus formas [3], entre los cuales se destacan los Hogares Comunitarios de Bienestar (HCB) tradicionales, pertenecientes al Instituto Colombiano del Bienestar Familiar (ICBF), que se extienden a todo el territorio nacional para trabajar en pro de los infantes buscando ofrecer un servicio de alta calidad. Sin embargo, muchos son los componentes que inciden en la percepción de la calidad del servicio por parte del usuario que resultan desfavorables, ya que no tienen un debido conocimiento y/o atención. Factores como: familia y comunidad; salud y nutrición; proceso pedagógico; talento humano; ambientes educativos y protectores; entornos educativos; y otros estándares [4]. Por ello, surgen herramientas estadísticas para estudiar dichos componentes con el propósito de aumentar la percepción del nivel de servicio de los usuarios, como es el caso del modelo SERVQUAL, que es una técnica de investigación comercial que permite realizar la medición de la calidad del servicio, conocer las expectativas de los clientes y cómo ellos aprecian el servicio [5]. Es una excelente opción para que las empresas revisen constantemente la percepción de sus clientes en cuanto al servicio que brinda.

En el municipio de Chibolo (Magdalena) existen $44 \mathrm{HCB}$ tradicionales que dan atención a 588 usuarias [6] laborando para el cumplimiento de los ejes estratégicos del Sistema Integrado de Gestión del ICBF [7], en donde la calidad, que es un eje de gran relevancia, necesita de un estudio estadístico de los componentes que inciden en la satisfacción del usuario dado que, actualmente, los estudios aplicados se han realizado en términos generales y parece ser que existen factores que están influyendo negativamente en las expectativas, percepción e importancia del servicio por parte del usuario. Lo anterior debido a que los conceptos de servicio y calidad se pueden asumir como factores diferenciadores de aquellas organizaciones que asumen su éxito desde la perspectiva de los clientes y referentes para elevar sus niveles de competitividad [8], partiendo como premisa desde la satisfacción de sus usuarios.

Por ello, se aplica el modelo estadístico SERVQUAL en las HBC tradicionales del municipio de Chibolo (Magdalena) con la finalidad de solucionar los problemas presentados en la percepción del servicio por parte de los usuarios debido a la falta de conocimiento referente a los componentes que inciden a la calidad de servicio. De esta manera, se ofrece a la comunidad investigativa una aplicabilidad de este modelo y, a las empresas, una forma de aumentar su competitividad.

\section{REVISIÓN LITERARIA}

\section{A. Análisis de fiabilidad}

Existen dos alternativas para llevar a cabo dicho análisis. La primera es mediante validación estadística, y la segunda, por la validación de expertos. De estas, la validación estadística es mayormente utilizada debido a su eficacia y existen varias herramientas para ejercerla. Un método efectivo es el coeficiente alfa de Cronbach que es un instrumento de medición y maneja valores entre 0-1. $\mathrm{Su}$ ventaja es que sus cálculos no precisan la división de los componentes a evaluar en dos mitades, simplemente se aplica la medición y se calcula el coeficiente [9].

La referencia [10] manifiesta que este coeficiente puede ser obtenido de dos maneras: i) mediante varianza de los ítems o ii) por medio de la matriz de correlación, teniendo en cuenta que el coeficiente alfa de Cronbach es aceptable con valor mínimo de 0,7 , y valores por debajo de este revelan una baja inconsistencia interna.

\section{B. Modelo SERVQUAL}

El modelo estadístico SERVQUAL se ha empleado para analizar la calidad de servicio dentro de muchas organizaciones a lo largo de los últimos 30 años. El cuestionario original ha sido analizado, criticado y adaptado a las necesidades y características de los diferentes sectores [11].

Es uno de los modelos de mayor aplicación actualmente en las organizaciones estadounidenses y fue desarrollado por sus autores Zeithaml, Parasuraman y Berry entre los años de 1985 y 1988 [12]. Este contiene las siguientes facetas:

1. Componentes del modelo SERVQUAL: estos pueden ser modificados según la finalidad del estudio y son las dimensiones en las que está sujeta dicho modelo. 
2. Expectativa y percepción: según la referencia [13], se entiende por expectativas el conjunto de creencias relacionadas con la prestación del servicio que funciona como estándares o puntos de referencias contra los cuales se juzga su desempeño, por lo que esta dimensión de la calidad resulta muy útil para el usuario al momento de realizar la evaluación de servicio y, según muchos autores, estas deben ser medidas.

Por otra parte, la percepción del cliente es la segunda dimensión de la calidad de servicio, y aunque esta varíe de acuerdo con la experiencia y al aprendizaje previo que tenga el observador, la referencia [14] la expresa como la sensación que experimenta un cliente después de recibir un producto o servicio. Esta se forma cuando éste evalúa el paquete de beneficios que le brinda la empresa, determinando costobeneficio y, luego, decide si la sensación es buena.

3. Grado de satisfacción: este se determina luego de efectuar una comparación entre las expectativas y perspectivas obtenidas de los clientes, lo cual se puede realizar mediante un estudio de gráfico en donde se observan las brechas existentes entre estas dimensiones, y cuyo análisis da a conocer si el nivel de satisfacción es relativamente bueno (normal), malo o bueno [15].

4. Grado de criticidad: se refiere a la situación en que los usuarios tienen altas expectativas en todos los componentes que no son satisfechos por la empresa, pero no representan un impacto significativo en la satisfacción general de los usuarios frente al servicio recibido.

\section{Calidad de servicio}

La entrega de servicios de calidad percibida deja de ser un diferencial y pasa a ser un imperativo para perpetuar y crecer en el mercado, siendo esencial para la implantación de estrategias [16].

Por ello la referencia [17] manifiesta que la calidad de servicio adquiere realidad en la percepción, considerando esta un deseo más que una percepción, ya que esta última implicaría un pensamiento y análisis anterior. Se tienen, así, 5 dimensiones globales: 1) tangibilidad, 2) confiabilidad, 3) tiempo de respuesta, 4) seguridad (confianza) y 5) empatía.

\section{Hogares Comunitarios de Bienestar}

El programa de Hogares Comunitarios de Bienestar (HCB) tradicional nace en el año 1986, se reglamenta en 1989 y se consolida cuando una madre comunitaria, en su casa, abre un espacio para atender entre 12 y 14 niños. Las madres o padres comunitarios son aquellos agentes educativos comunitarios responsables del cuidado de los niños y las niñas de primera infancia del programa de Hogares Comunitarios de Bienestar. Son reconocidos en su comunidad por su solidaridad, convivencia y compromiso con el desarrollo de los niños, niñas y sus familias [3].

\section{Metodología}

Este proyecto presenta un enfoque de tipo cuantitativo debido a que pretende evaluar y medir un fenómeno en particular basándose en el uso de técnicas estadísticas en medio de un proceso secuencial, deductivo y probatorio con el cual se pretende llegar a una serie de conclusiones. Además, su alcance es de tipo descriptivo, debido a que considera al fenómeno en estudio y sus componentes, mide los conceptos y define las variables necesarias [18].

Así mismo, se tienen como variables dependientes: familia y comunidad; salud y nutrición; proceso pedagógico; talento humano; ambientes educativos y protectores; y entornos educativos. Además, se cuentan con 37 estándares de calidad [4] que permiten la operacionalización de las variables.

La ecuación (1) fue utilizada para obtener una muestra (n) de tamaño 46 usuarios con los cuales se infirieron los resultados de una población (N) de 588 usuarios pertenecientes a las 44 HBC-Tradicionales, con un nivel de confianza del $95 \%$ y un error de muestreo de 0,1, y de los resultados de la referencia [7] se deduce que la proporción de usuarios satisfechos es de 0,85 .

$$
\boldsymbol{n}=\frac{\frac{z^{2} P * Q}{e^{2}}}{\frac{N-1}{N}+\frac{1}{N}\left(\frac{z^{2} P * Q}{e^{2}}\right)}=\frac{\frac{1,96^{2} * 0,85 * 0,15}{0,1}}{\frac{587}{588}+\frac{1}{588}\left(\frac{1,96^{2} * 0,85 * 0,15}{0,1^{2}}\right)} \approx \mathbf{4 6}(1)
$$

Para la aplicación del modelo SERVQUAL al caso de estudio, se hizo uso de una metodología efectiva compuesta mediante una relación lógica, la cual consta de las siguientes fases:

\section{A. Fase I: aplicación de técnicas estadísticas para la recolección de información}

Esta contiene las siguientes actividades para su cumplimiento:

\section{1) Diseño de cuestionario}

En este se efectúa una estructura que permita delimitar el modelo estadístico propuesto a unos lineamientos que faciliten alcanzar la investigación, teniendo en cuenta los componentes de calidad necesarios. Esta se divide en dos secciones, en la primera, se evalúa la relevancia de los componentes decalidad, y en la segunda, se avalan las expectativas y percepciones de los estándares de calidad.

\section{2) Recolección y tabulación de la información}

Para ello se hace uso de un método estadístico apropiado, como el muestreo aleatorio simple (MAS) [20], que permita realizar la encuesta a la muestra determinada y, luego, se tabula digitalmente para facilitar su visualización y organización. 


\section{3) Análisis de fiabilidad}

Se utiliza la validación estadística del coeficiente de Cronbach para conocer la confiabilidad de la medición. Para su cálculo, se utiliza el programa SPSS debido a su efectividad.

B. Fase II: evaluación de la expectativa, la importancia y la percepción de los usuarios frente a los componentes y dimensiones de calidad

\section{1) Evaluación de la importancia de cada componente de calidad}

Se realiza la codificación de los componentes con la finalidad de conocer el grado de importancia que le atribuyen los usuarios a la calidad de servicio y construir el modelo SERVQUAL (Fig. 1).

\section{2) Evaluación de la expectativa de los usuarios:}

Aquí se realiza un análisis numérico de las valoraciones obtenidas en relevancia a las expectativas de los usuarios frente a los estándares de calidad.

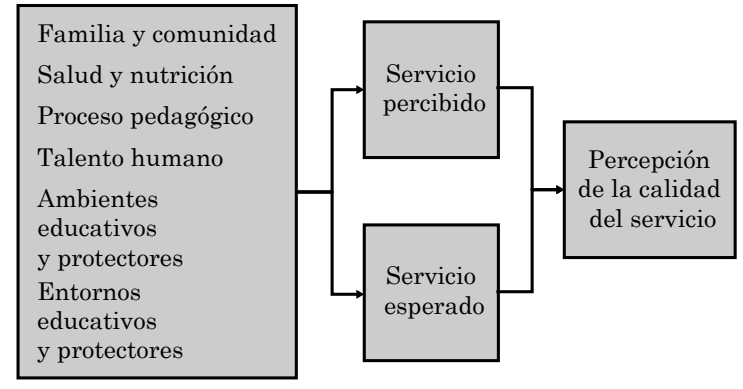

Fig. 1. Estructura del modelo SERVQUAL adaptado. Fuente: modificación de [19].

\section{3) Evaluación de la percepción de los usuarios}

$\mathrm{Al}$ igual que el caso anterior, se busca valorar numéricamente, mediante un análisis visual por métodos de recopilación, la percepción de los usuarios frente a los estándares de calidad.

C. Determinación de los componentes de calidad que inciden de forma crítica en la satisfacción de usuarios

\section{1) Identificación del grado de satisfacción} (GS) de los usuarios por componente

Se pretende conocer las brechas o distancias entre la percepción (Per) y la expectativa (Exp). Para, posteriormente, obtener el grado de satisfacción, lo cual se obtiene matemáticamente con (2).

$$
\boldsymbol{G S}=\operatorname{Per}-\operatorname{Exp}
$$

\section{2) Identificación del grado de criticidad (GC) por componente}

Este nos ayuda a conocer el nivel de afectación que tienen los componentes frente al servicio afectado, lo cual se puede calcular matemáticamente por medio de (3).

$$
\boldsymbol{G C}=G S * \operatorname{Imp}
$$

3) Determinación del componente de calidad crítico para la satisfacción de los usuarios del servicio

Aquí se obtienen las conclusiones referentes al grado de criticidad asignado por componente, lo cual se puede conseguir haciendo uso de las siguientes consideraciones:

Componente muy crítico: son los componentes que afectan altamente la percepción de calidad por parte de los usuarios frente al servicio prestado por la empresa. Se considerarán en este grado de criticidad aquellos componentes que presenten un coeficiente mayor que 19.

Componente crítico: son los componentes que afectan medianamente la percepción de calidad por parte de los usuarios frente al servicio prestado por la empresa. Se considerarán en este grado de criticidad a aquellos componentes que presenten un coeficiente entre 10 y 19.

Componente con bajo grado de criticidad: son los componentes que afectan en menor medida la percepción de calidad por parte de los usuarios frente al servicio prestado por la empresa. Se considerarán en este grado de criticidad a aquellos componentes que presenten un coeficiente menor que 10

\section{Resultados}

\section{A. Aplicación de técnicas estadísticas para la recolección de información.}

\section{1) Diseño de cuestionario}

La estructura del cuestionario se basa en una adaptación del modelo SERVQUAL para que contemple el cumplimiento del objetivo de la investigación, por lo que en este caso se tomarán como referencia los 6 componentes y los 36 estándares de calidad observados en el manual operativo de los hogares infantiles de Chibolo, Magdalena [4].

El cuestionario contempla dos secciones:

Sección 1: evaluación de la importancia de los componentes de calidad (codificación de la sección = EI).

En esta sección se desea evaluar la importancia de cada componente de calidad, por lo cual, en el cuestionario, los usuarios tienen la opción de asignarle un peso relativo (en porcentaje de importancia) a cada uno, teniendo en cuenta que la suma total debe dar como resultado el $100 \%$. 
Se codifican las preguntas y respuestas de la siguiente manera para los análisis estadísticos posteriores: EI-FC, EI-SN, EI-PP, EI-TH, EI-AE, EI-EE.

Sección 2: evaluación de expectativas y percepción de calidad en las situaciones descritas (codificación de la sección EE y EP).

En esta sección se pretende evaluar la expectativa y percepción que tiene el usuario frente a las dimensiones de calidad contempladas en el manual operativo de la empresa, por lo cual, el encuestado tiene como opciones de respuesta una escala de 1 al 3 , en donde 1 representa el puntaje más bajo y 3 el puntaje más alto.

Para evaluar la expectativa del encuestado frente a cada situación se codificarán las opciones de la siguiente manera:

$1=$ No es totalmente esencial

$2=$ Neutro o indiferente

$3=$ Definitivamente esencial

Para evaluar la percepción del encuestado frente a cada situación se codificarán las opciones de la siguiente manera:

$1=$ Totalmente en desacuerdo

$2=\mathrm{Ni}$ de acuerdo, ni en desacuerdo

$3=$ Totalmente de acuerdo

\section{2) Recolección y tabulación de la información}

Para la recolección de la información se aplica una encuesta a la muestra seleccionada de 46 usuarias de HBC tradicional del municipio de Chibolo, lo cual se lleva a cabo por el método MAS en donde se enumera la población (588 usuarios), posteriormente, se generan números aleatorios en ese rango y se obtienen los 46 números aleatorios (usuarios) que indica el tamaño de muestra. Esto debido a la dificultad que representa llevar a cabo un censo sobre la población en estudio.

Los investigadores principales se desplazaron al lugar de ejecución de las pruebas para dirigirse cada uno hacia las direcciones de los usuarios que comprenden la muestra; en cada lugar se procedió a socializar brevemente el objetivo de la investigación y explicar el cuestionario. Así mismo, ellos se encargarán de la recolección y evaluación física de los cuestionarios ejecutados.

Finalmente, la información obtenida en la ejecución de los cuestionarios será transcrita y tabulada virtualmente para facilitar el tratamiento de los datos en el estudio.

\section{3) Análisis de fiabilidad}

Al obtener los datos de las pruebas, los cuales se pueden observar en los resultados de las encuestas desarrolladas por los usuarios pertenecientes a la muestra, se procede a analizarlos a través del método alfa de Cronbach para validar la confiabilidad del instrumento de medición [9].
Tabla 1. Análisis de fiabilidad.

\begin{tabular}{|c|c|c|}
\hline $\begin{array}{c}\text { Alfa de } \\
\text { Cronbach }\end{array}$ & $\begin{array}{c}\text { Alfa de Cronbach basada en } \\
\text { elementos estandarizados }\end{array}$ & $\begin{array}{c}\text { N de } \\
\text { elementos }\end{array}$ \\
\hline 0,883 & 0,850 & 74 \\
\hline
\end{tabular}

Fuente: elaboración propia.

En la Tabla 1 se puede apreciar que el alfa de Cronbach de los elementos en estudio es de 0,883, y por ser un valor mayor a 0,8 se puede afirmar que las preguntas son congruentes entre sí, y, por ende, aportan a la encuesta.

\section{B. Evaluación de la importancia, expectativa y percepción de los usuarios frente a los componentes y dimensiones de calidad}

\section{1) Evaluación de la importancia de cada componente de calidad}

En el modelo SERVQUAL adaptado a las necesidades del estudio, se indica, en primera instancia, la evaluación de la importancia que le atribuyen los usuarios a cada uno de los componentes de calidad preestablecidos.

Para analizar los datos resultantes en adelante, se debe tener en cuenta que $\mathrm{FC}=$ familia $\mathrm{y}$ comunidad, $\mathrm{SN}=$ salud y nutrición, $\mathrm{PP}=$ proceso pedagógico, $\mathrm{TH}=$ talento humano, $\mathrm{AE}=$ ambientes educativos y protectores, $\mathrm{EE}=$ entornos educativos y protectores.

Los valores medios asignados por los usuarios en la evaluación de importancia de los componentes de calidad son entonces los siguientes:

TAbla 2. Evaluación media de la importancia.

\begin{tabular}{|c|c|c|c|c|c|}
\hline FC & SN & PP & TH & AE & EE \\
\hline 18,35 & 31,24 & 12,72 & 17,91 & 10,02 & 9,80 \\
\hline
\end{tabular}

Fuente: elaboración propia.

Dadas las condiciones anteriormente descritas, se puede concluir que, a $\mathrm{FC} \mathrm{y} \mathrm{TH}$, en promedio, los usuarios los evalúan con un grado medio de importancia; por su parte, PP, AE y EE, en promedio, se evalúan con un grado bajo de importancia. Por último, se destaca que el componente salud $y$ nutrición, en promedio, es evaluado con un grado alto de importancia.

\section{2) Evaluación de la expectativa de los usuarios}

Prosiguiendo con el proceso metodológico descrito, en segunda instancia, se busca realizar un análisis de la evaluación asignada por parte de los usuarios teniendo en cuenta sus expectativas frente a cada estándar de calidad. 
En favor de visualizar de mejor manera el análisis, se contemplan los resultados de la evaluación media de expectativas de los usuarios frente a los estándares de calidad por cada componente.

Componente FC

Tabla 3. Evaluación media de la expectativa FC

\begin{tabular}{|c|c|c|c|c|c|c|c|c|}
\hline S1 & S2 & S3 & S4 & S5 & S6 & S7 & S8 & S9 \\
\hline 2,93 & 2,57 & 2,67 & 2,80 & 2,93 & 2,89 & 2,72 & 2,70 & 2,70 \\
\hline
\end{tabular}

Fuente: elaboración propia.

Por lo tanto, la expectativa media de los usuarios frente al componente FC es de 2,77.

Componente SN

Tabla 4. Evaluación media de La expectativa SN

\begin{tabular}{|l|l|l|l|l|l|l|l|l|}
\hline $\mathrm{S} 10$ & $\mathrm{~S} 11$ & $\mathrm{~S} 12$ & $\mathrm{~S} 13$ & $\mathrm{~S} 14$ & $\mathrm{~S} 15$ & $\mathrm{~S} 16$ & $\mathrm{~S} 17$ & $\mathrm{~S} 18$ \\
\hline 3,00 & 2,91 & 3,00 & 3,00 & 2,89 & 2,87 & 2,96 & 2,85 & 2,85 \\
\hline
\end{tabular}

Fuente: elaboración propia.

Por lo tanto, la expectativa media de los usuarios frente al componente SN es de 2,92.

Componente PP

Tabla 5. Evaluación media de la expectativa PP

\begin{tabular}{|c|c|c|c|}
\hline $\mathrm{S} 19$ & $\mathrm{~S} 20$ & $\mathrm{~S} 21$ & $\mathrm{~S} 22$ \\
\hline 2,96 & 2,98 & 2,93 & 2,74 \\
\hline
\end{tabular}

Fuente: elaboración propia.

Por lo tanto, la expectativa media de los usuarios frente al componente PP es de 2,90.

Componente $\mathrm{TH}$

Tabla 6. Evaluación media de la expectativa TH

\begin{tabular}{|l|l|l|}
\hline $\mathrm{S} 23$ & $\mathrm{~S} 24$ & $\mathrm{~S} 25$ \\
\hline 3,00 & 2,43 & 2,70 \\
\hline
\end{tabular}

Fuente: elaboración propia.

Por lo tanto, la expectativa media de los usuarios frente al componente TH es de 2,71.

Componente AE

Tabla 7. Evaluación media de la expectativa AE

\begin{tabular}{|c|c|c|c|c|c|c|c|}
\hline S26 & S27 & S28 & S29 & S30 & S31 & S32 & S33 \\
\hline 3,00 & 2,91 & 2,89 & 3,00 & 2,93 & 2,87 & 2,59 & 2,87 \\
\hline
\end{tabular}

Fuente: elaboración propia.

Por lo tanto, la expectativa media de los usuarios frente al componente AE es de 2,88.

\section{Componente EE}

Tabla 8. Evaluación media de la expectativa EE

\begin{tabular}{|l|l|l|l|}
\hline S34 & S35 & S36 & S37 \\
\hline 2,98 & 2,85 & 2,96 & 2,63 \\
\hline
\end{tabular}

Fuente: elaboración propia.

Por lo tanto, la expectativa media de los usuarios frente al componente EE es de 2,85.

Al final, se puede resumir la evaluación de la expectativa de la siguiente manera (Fig. 2):

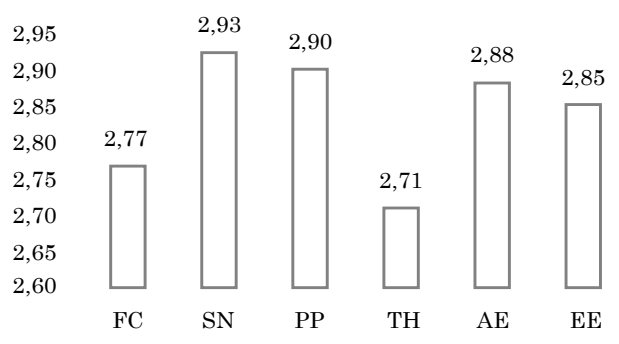

Fig. 2. Evaluación de la expectativa de los usuarios. Fuente: elaboración propia.

\section{1) Evaluación de la percepción de los usuarios}

En tercera instancia se sigue con el proceso metodológico planteado, se busca realizar un análisis de la evaluación asignada por parte de los usuarios teniendo en cuenta sus percepciones frente a los estándares de calidad.

En favor de visualizar de mejor manera el análisis, se contemplan los resultados de la evaluación de percepciones de los usuarios frente a las situaciones planteadas por cada componente.

Componente FC

Tabla 9. Evaluación Media de la Percepción FC

\begin{tabular}{|c|c|c|c|c|c|c|c|c|}
\hline $\mathrm{S} 1$ & $\mathrm{~S} 2$ & $\mathrm{~S} 3$ & $\mathrm{~S} 4$ & $\mathrm{~S} 5$ & $\mathrm{~S} 6$ & $\mathrm{~S} 7$ & $\mathrm{~S} 8$ & $\mathrm{~S} 9$ \\
\hline 1,67 & 1,65 & 2,22 & 1,59 & 1,80 & 2,02 & 2,11 & 1,70 & 1,74 \\
\hline
\end{tabular}

Fuente: elaboración propia.

Por lo tanto, la percepción media de los usuarios frente al componente FC es de 1,83.

Componente SN

Tabla 10. Evaluación Media de la Percepción SN

\begin{tabular}{|l|l|l|l|l|l|l|l|l|}
\hline $\mathrm{S} 10$ & $\mathrm{~S} 11$ & $\mathrm{~S} 12$ & $\mathrm{~S} 13$ & $\mathrm{~S} 14$ & $\mathrm{~S} 15$ & $\mathrm{~S} 16$ & $\mathrm{~S} 17$ & $\mathrm{~S} 18$ \\
\hline 2,17 & 1,83 & 1,85 & 2,76 & 1,89 & 2,26 & 2,04 & 2,17 & 1,93 \\
\hline
\end{tabular}

Fuente: elaboración propia.

Por lo tanto, la percepción media de los usuarios frente al componente SN es de 2,10. 


\section{Componente PP}

Tabla 11. Evaluación Media de la Percepción PP

\begin{tabular}{|c|c|c|c|}
\hline S19 & S20 & S21 & S22 \\
\hline 1,85 & 2,37 & 2,30 & 1,39 \\
\hline
\end{tabular}

Fuente: elaboración propia.

Por lo tanto, la percepción media de los usuarios frente al componente PP es de 1,98.

Componente $\mathrm{TH}$

Tabla 12. Evaluación Media de la Percepción TH

\begin{tabular}{|c|c|c|}
\hline S23 & S24 & S25 \\
\hline 2,41 & 1,74 & 1,89 \\
\hline
\end{tabular}

Fuente: elaboración propia.

Por lo tanto, la percepción media de los usuarios frente al componente $\mathrm{TH}$ es de 2,01 .

Componente AE

Tabla 13. Evaluación Media de la Percepción AE

\begin{tabular}{|c|c|c|c|c|c|c|c|}
\hline S26 & S27 & S28 & S29 & S30 & S31 & S32 & S33 \\
\hline 2,28 & 2,30 & 2,24 & 2,59 & 2,22 & 1,72 & 1,46 & 1,52 \\
\hline
\end{tabular}

Fuente: elaboración propia.

Por lo tanto, la percepción media de los usuarios frente al componente AE es de 2,04.

Componente EE

Tabla 14. Evaluación Media de la Percepción EE

\begin{tabular}{|c|c|c|c|}
\hline S34 & S35 & S36 & S37 \\
\hline 1,98 & 1,83 & 1,96 & 1,65 \\
\hline
\end{tabular}

Fuente: elaboración propia.

Por lo tanto, la percepción media de los usuarios frente al componente FC es de 1,85.

Al final, se puede resumir la evaluación de la percepción de los usuarios de la siguiente manera (Fig. 3):

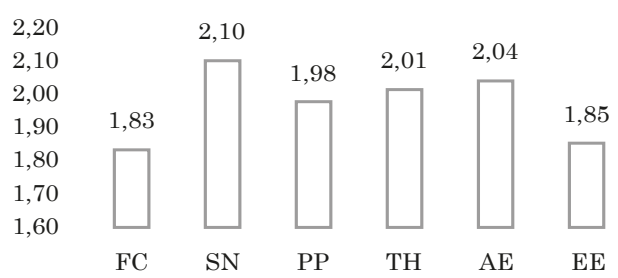

Fig. 3. Evaluación de la percepción de los usuarios. Fuente: elaboración propia.
C. Determinación de los componentes de calidad que inciden críticamente en la satisfacción de los usuarios

\section{1) Identificación del grado de satisfacción de los usuarios por componente}

En esta etapa, en primera instancia, se busca determinar las distancias o brechas existentes entre la expectativa de los usuarios y su percepción frente al servicio recibido.

Se puede analizar que existen brechas evidentes en todos los componentes de calidad, lo cual quiere decir que, para los clientes, el servicio prestado por la empresa no cumple con sus expectativas (Fig. 4).

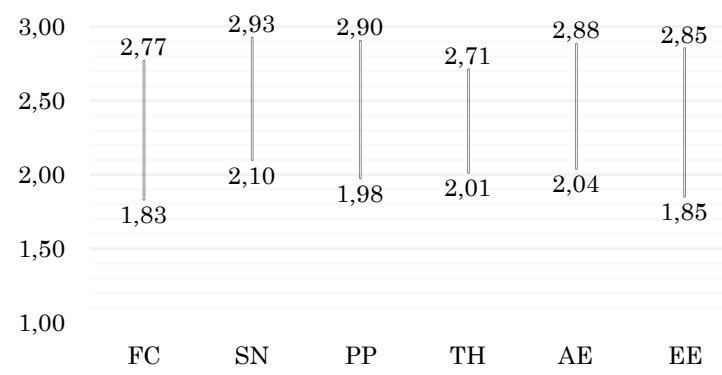

Fig. 4. Brechas en el servicio. Fuente: elaboración propia.

Para determinar el grado de satisfacción de cada componente se utiliza (2) y se obtienen los siguientes resultados (Fig. 5).

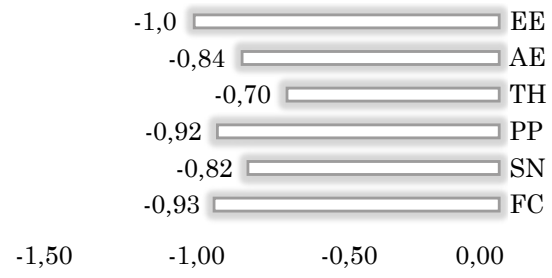

Fig. 5. Grado de satisfacción de los usuarios. Fuente: elaboración propia.

Al analizar la Fig. 5, se logra determinar que ningún componente logra satisfacer a los usuarios, destacándose los componentes EE, FC y PP como los que generan mayor grado de insatisfacción.

\section{2) Identificación del grado de criticidad por componente}

A pesar de existir brechas en todos los componentes, estas no representan una distancia tan amplia entre las expectativas y percepciones por parte de los usuarios. Esto se debe al componente "grado de criticidad".

Para realizar el cálculo del grado de criticidad se recurre a (3), y se obtienen los resultados que se reflejan en la Tabla 15. 
Tabla 15. Grado de criticidad Por COMPonente

\begin{tabular}{|c|c|c|c|c|c|}
\hline Componente & Exp. & Per. & $\begin{array}{c}\text { G. } \\
\text { Sat. }\end{array}$ & Imp. & $\begin{array}{c}\text { G. } \\
\text { Criticidad }\end{array}$ \\
\hline FC & 2,77 & 1,83 & $-0,93$ & 18,35 & 17,15 \\
\hline SN & 2,93 & 2,10 & $-0,82$ & 31,24 & 25,73 \\
\hline $\mathrm{PP}$ & 2,90 & 1,98 & $-0,92$ & 12,72 & 11,75 \\
\hline $\mathrm{TH}$ & 2,71 & 2,01 & $-0,70$ & 17,91 & 12,46 \\
\hline $\mathrm{AE}$ & 2,88 & 2,04 & $-0,84$ & 10,02 & 8,44 \\
\hline $\mathrm{EE}$ & 2,85 & 1,85 & $-1,00$ & 9,80 & 9,80 \\
\hline
\end{tabular}

Fuente: elaboración propia.

\section{3) Determinación de componentes de calidad críticos para la satisfacción de los usuarios del servicio}

El grado de criticidad atribuido a cada componente se detalla en la siguiente figura (Fig. 6):

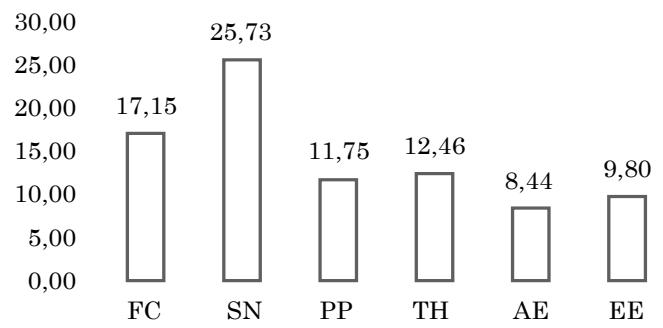

Fig. 6. Grado de criticidad por componente. Fuente: elaboración propia.

Se puede determinar entonces que el componente $\mathrm{SN}$ es el que genera mayor impacto en la insatisfacción de los usuarios frente al servicio prestado en los hogares infantiles de Chibolo, Magdalena, debido a que es aquel que tiene un mayor grado de criticidad, categorizándose además como un componente crítico por obtener una calificación mayor que 19 .

Por su alto grado de criticidad, corresponde realizar un análisis del grado de satisfacción de los clientes frente a cada uno de los estándares de calidad del componente $\mathrm{SN}$, de lo cual resulta la tabla 16 .
Así mismo, se puede decir que los componentes FC, TH y PP son componentes con un grado de criticidad medio, puesto que obtuvieron una calificación inmersa en el rango entre 10 y 19.

Por último, se deduce que los componentes $\mathrm{AE} \mathrm{y}$ EE son los menos influyentes en la satisfacción de los usuarios debido a que tuvieron calificaciones menores a 10 , por lo cual, se categorizan como componentes con bajo grado de criticidad.

\section{Conclusiones}

Como resultado de este proyecto investigativo se logró determinar que el componente salud y nutrición (SE) es el que incide de forma crítica en la satisfacción de los usuarios en los hogares infantiles de Chibolo, Magdalena; por lo cual es fundamental realizar un estudio detallado en cuanto a cada una de los estándares de calidad que lo integran para reducir la brecha existente entre la expectativa y percepción, generando de esta forma un impacto significativo en el grado de satisfacción.

En este caso, los denominados estándares 11, 12 y 14 en el cuestionario deben ser el foco de atención debido a que específicamente son los que están contribuyendo en mayor magnitud a la insatisfacción de los usuarios.

Para dar cumplimiento y mejorar la percepción de calidad frente a los estándares detectados, se debe, entonces, incentivar la realización de actividades educativas y formativas en hábitos de alimentación y salud que involucren tanto a los beneficiarios como a los usuarios, además, proporcionar complementación alimentaria adecuada y adaptar la minuta patrón a las necesidades alimentarias de los beneficiarios.

Es recomendable efectuar un seguimiento a este componente para evaluar su efecto en la satisfacción de los usuarios a través del tiempo. Por esto, resulta indispensable manejar un plan de seguimiento frecuente a través de indicadores de calidad en cada uno de los componentes de la calidad del servicio, brindando atención especial a aquellos considerados como más críticos.

Por último, se destaca que el presente artículo contribuye a futuras investigaciones como documento de consulta teórica y metodológica práctica para el estudio de los factores que inciden en la satisfacción de los usuarios frente a la prestación de un determinado servicio brindado por una empresa en particular.

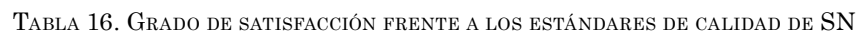

\begin{tabular}{|c|c|c|c|c|c|c|c|c|c|}
\hline $\mathrm{S}$ & $\mathrm{S} 10$ & $\mathrm{~S} 11$ & $\mathrm{~S} 12$ & $\mathrm{~S} 13$ & $\mathrm{~S} 14$ & $\mathrm{~S} 15$ & $\mathrm{~S} 16$ & $\mathrm{~S} 17$ & $\mathrm{~S} 18$ \\
\hline $\mathrm{E}$ & 3,00 & 2,91 & 3,00 & 3,00 & 2,89 & 2,87 & 2,96 & 2,85 & 2,85 \\
\hline $\mathrm{P}$ & 2,17 & 1,83 & 1,85 & 2,76 & 1,89 & 2,26 & 2,04 & 2,17 & 1,93 \\
\hline $\mathrm{GS}$ & $-0,83$ & $-1,09$ & $-1,15$ & $-0,24$ & $-1,00$ & $-0,61$ & $-0,91$ & $-0,67$ & $-0,91$ \\
\hline
\end{tabular}

Fuente: elaboración propia. 


\section{Financiación}

Este artículo de investigación se deriva del proyecto titulado "Componentes de la calidad que inciden en la satisfacción de los usuarios del servicio prestado por una empresa a niños y niñas entre 2 y 5 años en el municipio de Chibolo" con el cual fue opción de grado para la especialización en estadística aplicada en la Universidad del Atlántico. El proyecto fue financiado por los autores y con apoyo de la Universidad del Atlántico por medio de tutorías y la posibilidad de acceso a recursos físicos y tecnológicos. Fecha de inicio: enero de 2017. Fecha de finalización: junio de 2017.

\section{REFERENCIAS}

[1] F. Marco, Calidad del cuidado y la educación para la primera infancia en América Latina. Santiago de Chile, Chile: Publicación de las Naciones Unidas, 2014, pp.1530 .

[2] Departamento Nacional de Planeación, "Primera Infancia”, 2016. [En línea]. Disponible en: https://www.dnp. gov.co/programas/desarrollo-social/pol\%C3\%ADticassociales-transversales/Paginas/primera-infancia.aspx [Recuperado: oct 09, 2017]

[3] Instituto Colombiano del Bienestar Familiar, "Primera infancia: Madres comunitarias", 2017 [En línea]. Disponible en: http://www.icbf.gov.co/portal/page/portal/PrimeraInfanciaICBF/Madres. [Recuperado: oct 11/10/2017]

[4] C. Plazas, "Manual operativo de la modalidad comunitaria para la atención a la primera infancia”, ICBF. Bogotá D.C, Colombia, Rep. Tec. MO15.PP, ene., 2016.

[5] R. Matsumoto, "Desarrollo del Modelo Servqual para la medición de la calidad del servicio en la empresa de publicidad Ayuda Experto" Rev. Perspectivas, vol. 17, no. 33, pp. 181-209, oct. 2017.

[6] C. De la Fuente, "Portafolio de servicios regional Magdalena Centro Zonal Plato Servicios de Educación inicial", ICBF. Plato (Magdalena), Colombia, Rep. Tec., 2016.

[7] C. Gutiérrez y A. Pulido, "Encuesta de satisfaccion de los usuarios del ICBF 2014”, Help File LTDA., Soledad, Colombia, Rep. Inf., 2014.

[8] M. E. Vargas y L. Aldana, Calidad y servicios: Conceptos y herramientas. Bogotá, Colombia: ECOE ediciones, 2015 , pp. $80-140$

[9] J. A. Bojórquez, L. López y E. Jiménez. "Utilización del alfa de Cronbach para validar la confiabilidad de un instrumento de medición de satisfacción del estudiante en el uso del software Minitab", LACCEI, Cancún, 2013, pp. 1-9.
[10] H. Celina y A. Campo, "Aproximación al uso del coeficiente alfa de Cronbach", Rev.Col.Psiquiat., vol. 35, no. 4, pp. 572-580, 2005.

[11] C. McCollin, I. Ograjensek y R. Gob, "SERVQUAL and the process improvement challenge", Quality and Reliability Engineering International, vol. 27, no. 5, pp. 705718, 2011. https://doi.org/10.1002/qre.1234

[12] V.Zeithmail, L. Berry y A. Parasuraman, "The nature and derterminants of customer expectations of service", Journal of the Academy of Marketing Science, vol. 21, no. 1, pp. 1-12, 1993. https://doi.org/10.1177/0092070393211001

[13] V. A. Zeithaml y M. J. Bitner, Marketing de servicios: un enfoque de integración del cliente a la empresa. México D.F, México: McGraw-Hill, 2002, pp. 747.

[14] E. Caldera, J. Pirela y E. Ortega, "Dimensiones para el estudio de la calidad de servicios en bibliotecas universitarias," Documentación de la Ciencias de la Información, vol. 34, pp. 333-347, 2011. https://doi.org/10.5209/ rev_DCIN.2011.v34.36462

[15] H. Pontón, "Medición de la satisfacción del cliente como parte de la calidad de servicio de los distribuidores de equipos y materiales para el sector de la publicidad exterior en el municipio Maracaibo," Rev. COEPTUM, vol. 1, no.1, pp. 1-6, oct. 2009

[16] T. Assunção, A. A. Queiroga, T.H. Bogoni y N. Rodrigues, "Qualidade em Serviços e Dispositivos Móveis: Uma Análise a Partir da Aplicação do Modelo Servqual," Rev. FSA, vol. 14 , no. 4 , p. 19-39, jul. 2017.

[17] L. Berry, D. R. Leonard y W. B. Carter, Calidad de servicios una ventaja estratégica para instituciones financieras, Madrid, España: Ediciones Díaz de los Santos, 1989, pp.90-120.

[18] R. Hernández, C. Fernández y M. P. Baptista, Metodología de la investigación. México D.F., México: McGRAWHILL, 2010, pp.2-22.

[19] E. J. Duque, "Revisión del concepto de calidad del servicio y sus modelos de medición", Innovar, vol. 15, no. 25, pp. $64-80$, jun. 2005 .

[20] D. Ospina, "Muestreo aleatorio simple", en Introducción al muestreo, 1era ed. Bogotá: Unibiblos, 2001, pp. $31-36$.

Víctor Enrique Valencia Espejo. Especialista en Estadística aplicada de la Universidad del Atlántico (2017). Graduado en Ingeniería Industrial de la Universidad del Magdalena (2016). https://orcid. org/0000-0002-2838-1218

Selenia Cruz Botto. Especialista en Estadística aplicada de la Universidad del Atlántico (2017). Graduada en Psicología de la Universidad del Magdalena (2012). http://orcid.org/0000-0002-7210-3186

Oscar José Ospino Ayala. Graduado en Ingeniería Industrial de la Universidad del Magdalena (2016). https://orcid.org/0000-0002-3876-6111 